\title{
MS28-04 | The Secret Life Of MOFs' Functional Groups: Should We Trust Random DISORDER?
}

Canossa, Stefano (Delft University of Technology, Delft); Gonzalez Nelson, Adrian (Delft University of Technology, Delft); Rega, Davide (Delft University of Technology, Delft); van der Veen, Monique (Delft University of Technology, Delft); Bürgi, Hans-Beat (Universität Bern, Bern, CH); Pei, Xiaokun (UC Berkeley, Berkeley, USA); Yaghi, Omar (UC Berkeley, Berkeley, USA)

The practice of endowing metal-organic frameworks (MOFs) with novel functionalities by adding chemical groups to their backbone is one of the key strategies to achieve better performances [1]. One of the simplest approaches in this regard is to employ functionalised ligands (linkers) in the final coordination network [2]. Since the functional groups often decrease the linkers' symmetry without affecting the framework connectivity, they usually occupy alternative positions with similar energy minima and thus occupancy values. Importantly, the assumption of their random distribution should be considered a misguided approximation as it excludes any relevant interaction between adjacent groups or with the framework itself. In this context we present the case studies of ZIF-90 [3] and $\mathrm{NO}_{2}-\mathrm{MIL}-53(\mathrm{Al}$ ) [4], whose linker disorder revealed to be strongly correlated or even crystallographic order upon symmetry breaking. Total scattering single crystal XRD analysis afforded corrected structural models showing important differences from the random disorder-based ones with consequences on relevant features such as pores opening, presence of dipole moments and availability of metal sites. These findings highlight the importance of local order of framework components in functionalised MOFs and the possible risks of trusting random disorder models.

[1] H. Furukawa et al., Science, 2013, 341, 1-12.

[2] W. Lu et al., Chem Soc Rev, 2014, 43, 5561-5593.

[3] O. M. Yaghi et al., J. Am. Chem. Soc., 2008, 130, 12626-12627.

[4] G. Férey et al., Chem. Commun., 2003, 0, 2976-2977. 